\title{
Editorial Message from the New Editor-in-Chief
}

Starting from 1983, ACM Transactions on Information Systems (TOIS) has provided a tremendous platform for the information retrieval (IR) research community to share ideas and progress on theories, algorithms, experiments, and applications on broad and various IR topics. Thanks go to the six past Editor-in-Chiefs (EiCs) who have devoted themselves to successfully leading the TOIS to be a top journal in the area with a highly regarded reputation. Thanks also go to our dozens of associate editors and hundreds of reviewers who have made great efforts as volunteers to provide fair, valuable, and detailed suggestions and comments and help the TOIS run healthily and efficiently. And thanks to the authors for your interest in this exceptional journal with highquality and high-impact work.

I am honored to serve as the new EiC of the leading journal TOIS since August 2020. Together with the dream team of the Editorial Board, we are excited and well-prepared for running the journal in a fair, effective, and efficient way. We will commit ourselves to keeping the journal's reputation and impacts, and fight for scientific improvements on IR research. We aim to contribute to the whole IR and other related communities by publishing solid and reproducible work, exploring future directions in the new age, and encouraging innovations across theory and applications.

Let me introduce the current Editorial Board:

\begin{tabular}{|c|c|}
\hline \multicolumn{2}{|l|}{ Senior Editors: } \\
\hline Tat-Seng Chua, & National University of Singapore \\
\hline Fabrizio Sebastiani & Italian National Research Council \\
\hline Tetsuya Sakai & Waseda University \\
\hline \multicolumn{2}{|l|}{ Editors: } \\
\hline Jaime Arguello & University of North Carolina at Chapel Hill \\
\hline Mark Carman & Polytechnic University of Milan, EU \\
\hline Hui Fang & University of Delaware \\
\hline Nicola Ferro & University of Padua \\
\hline Jiafeng Guo & Chinese Academy of Science \\
\hline Xiangnan $\mathrm{He}$ & University of Science and Technology of Chin \\
\hline Zi (Helen) Huang & The University of Queensland \\
\hline Evangelos Kanoulas & University of Amsterdam \\
\hline Chenliang Li & Wuhan University \\
\hline Hang Li & ByteDance Technology \\
\hline Vanessa Murdock & Amazon \\
\hline Iadh Ounis & Glasgow University \\
\hline Benjamin Piwowarski & French National Centre for Scientific Research \\
\hline Suzan Verberne & Leiden University \\
\hline Yang Wang & Hefei University of Technology \\
\hline
\end{tabular}

ACM Reference format:

Min Zhang. 2021. Editorial Message from the New Editor-in-Chief. ACM Trans. Inf. Syst. 39, 3, Article 23e (June 2021), 2 pages.

https://doi.org/10.1145/3447945

(C) 2021 Association for Computing Machinery.

1046-8188/2021/06-ART23e \$15.00

https://doi.org/10.1145/3447945 

Lexing Xie
Emine Yilmaz
Hamed Zamani
Yongfeng Zhang

\author{
Australian National University \\ University College London, Amazon \\ University of Massachusetts Amherst \\ Rutgers University
}

I would like to share my visions for TOIS in its 40's and the future:

Activity and Efficiency: No matter how old TOIS is, it is always an active journal that meets the dynamics of the era and the field. We make functional interactions within the Editorial Board with frequent discussions and half-year regular meetings. We encourage the engagement of the community and connection with related conferences. For example, TOIS accepted papers also give presentations on the year's Special Interest Group on Information Retrieval conference (SIGIR) to increase interactions and impacts. We are open to and tracing the challenges, trends, changes, and future directions of the IR field. We always emphasize the fast process of submissions. Although the number of submissions is significantly increasing (+67\% in 2020), the average time from submission to the first decision has decreased from 46 days to 39 days in 2020, even in the severe pandemic period when everyone faces unprecedented busy schedules.

Fairness and Diversity: Fairness is one of the significant principles of academic publications. We keep it as the gold standard in processing TOIS manuscripts. The organization of the Editorial Board is diverse in terms of region and research topic; gender and academia/industry are also taken into consideration and will be enhanced continuously. We are working to build an adequate pool of trusted reviewers who could help provide fair, qualified, detailed, and effective comments efficiently. More paper types will be encouraged, such as academic and industry submissions, survey papers, and perspective papers.

Quality and Reproducibility: Quality is the critical metric that we insist on, making TOIS the top journal. We pay attention to both the publications' quality and the reviews' quality, which benefit the authors whether their work is accepted or not. Meanwhile, as emphasized by the SIGIR community in recent years, reproducibility is also an essential issue for the journal paper. It will be noted to the authors (for the applicable manuscript types) and considered in the reviewing process.

If you have any suggestions to strengthen TOIS at any point, please feel free to contact me. And you are warmly welcome to contribute to TOIS by submissions, reviews, editorial tasks, and discussions. Let's try our best to improve the IR community for a bright future together.

Min Zhang

Tsinghua University ACM TOIS Editor-in-Chief 